\title{
SECADO A LA INTEMPERIE MEDIANTE APILADO EN CABALLETES Y CUADRADOS HORIZONTALES DE FERNANSÁNCHEZ (Triplaris cumingiana Fish. Mey.) Y LAUREL (Cordia alliodora Ruiz \& Pav.) EN EL CANTON QUEVEDO, ECUADOR
}

\author{
Fidel Antonio Troya Mera ${ }^{1}$, José Pedro Suatunce Cunuhay ${ }^{1,2}$, Guillermo Law Blanco ${ }^{2}$ \\ ${ }^{1}$ Unidad de Investigación Científica y Tecnológica, Universidad Técnica Estatal de Quevedo, $\mathrm{km} 1$ 11/2 \\ vía Quevedo - Santo Domingo de los Tsáchilas, C. P. 73. Quevedo, Los Ríos, Ecuador. \\ ${ }^{2}$ Facultad de Ciencias Ambientales, Universidad Técnica Estatal de Quevedo, $\mathrm{km} 1$ 11/2 vía a Santo Domingo \\ de los Tsáchilas, C. P. 73. Quevedo, Los Ríos, Ecuador.
}

\begin{abstract}
Resumen
Se evaluaron durante cinco meses, defectos de secado de la madera: abarquillados, combados, torceduras, rajaduras y grietas superficiales; así como también el contenido de equilibrio de humedad en las maderas de fernansánchez ( $T$. cumingiana) y laurel (C. alliodora). Se compararon dos métodos de apilamiento: caballetes y cuadrados horizontales, en el secado de tablas de tres secciones del árbol (basal, media y terminal). No hubo diferencias significativas entre los métodos de apilamiento ni entre las secciones del árbol. Se determinó que es suficiente con 90 días de secado a la intemperie para que la madera de ambas especies alcance el contenido de humedad de equilibrio de la zona donde se realizó la investigación.
\end{abstract}

Palabras claves: secado natural, alabeos, rajaduras, grietas superficiales, contenido de humedad, Triplaris cumingiana Fish. Mey., Cordia alliodora Ruiz \& Pav
During five months wood drying defects were evaluated: curled up, warped, twist, cracks, superficial fissures; as well as the moisture content balance in the fernansánchez (T. cumingiana) and laurel (C. alliodora) woods. Two piling up methods were compared: easels and horizontal squares, in the drying of boards of three sections of the tree (basal, middle and terminal). There were not significant differences between the piling up methods neither among the sections of the tree. It was determined that it is enough with 90 days of outdoors drying for the wood of both species to reach the moisture content balance of the area where the investigation was carried out.

Key words: natural drying, warps, cracks, superficial fissures, content of humidity, Triplaris cumingiana Fish. Mey., Cordia alliodora Ruiz \& Pav.

\section{INTRODUCCIÓN}

$\mathrm{E}^{1}$ objetivo primordial del secado de la madera es obtener un producto sin defectos ni alteraciones, que pueda seguir transformándose sin causar problemas derivados. Con el secado se consigue establecer un equilibrio higroscópico entre el medio ambiente y la madera. Este proceso es de mucha importancia cuando no se cuenta con secadores artificiales debido a que es un paso imprescindible antes de empezar a trabajar la madera. El correcto secado es uno de los primeros retos a los que deben enfrentarse quienes vayan a trabajar con este material y quieran competir por la calidad (Fernández, 1998). El contenido de equilibrio de humedad es un factor muy importante en lo que tiene que ver con el uso de la madera. De él depende una buena parte de sus propiedades físicas y mecánicas, y su estabilidad

Recibido: Junio, 2008. Aceptado: Enero, 2009.

Publicado como ARTÍCULO en Ciencia y Tecnología 3: 35-39. 2009. dimensional, así como también su resistencia al ataque de hongos e insectos, y mayor o menor facilidad para ser trabajada (Orbe, 1989).

La humedad de la madera influye en los numerosos procesos de transformación. Así, el agua se interpone entre la madera, la cola o el barniz, dificultando la realización de estos procesos. Pero más importante que esta acción está el hecho de que al variar el contenido de humedad de la madera, varían sus dimensiones (Peña, 2000). Cuando la madera experimenta un secado brusco, normalmente se deforma, ya que la pérdida de humedad no es moderada, sino abrupta; esto altera su constitución anatómica y ocasiona defectos tales como alabeos (abarquillado, combado, torcedura), rajaduras y grietas (COPANT, 1969).

Existen diversas maneras de apilar la madera para el secado: al aire libre o bajo cubierta; entre ellas 
están las disposiciones en caballetes y cuadrados horizontales. Estas formas de apilamiento permiten la circulación del aire entre las tablas; además, si el apilado de las piezas es correcto, se reduce el nivel de deformaciones (Junta del Acuerdo de Cartagena, 1989).

El fernansánchez y el laurel son dos especies forestales cuyas maderas son semiduras y en nuestro medio son utilizadas en mueblería, puertas, pisos, carpintería, chapas, revestimientos, parquet, embalaje, encofrado y construcciones (Junta del Acuerdo de Cartagena 1981, OIMT 2001). Debido a que la información sobre secado natural de estas especies, es muy escasa en nuestro medio, este artículo provee datos útiles sobre las diferencias y semejanzas en el secado natural, al apilar la madera de laurel y fernansánchez en caballetes y cuadrados horizontales. Esta información orientará a los artesanos (ebanistas y carpinteros) en la realización de mejores trabajos.

El objetivo de este estudio fue evaluar y comparar las deformaciones (alabeos, rajaduras y grietas superficiales) en la madera de Triplaris guayaquilensis (fernansánchez) y Cordia alliodora (laurel) sometida al secado a la intemperie, durante la época seca; así como también determinar mensualmente el contenido de humedad, con el propósito de observar las fechas en que la madera de las especies en estudio alcanza el contenido de humedad de equilibrio $(18,8 \%)$ en el área experimental.

\section{Materiales y Métodos}

$\mathrm{L}$ a investigación se realizó en la Zona Central del Litoral ecuatoriano, en el cantón Quevedo, recinto "La Piragua" Finca "Rosita", ubicado entre las coordenadas $79^{\circ} 25^{\prime} 18^{\prime \prime}$ de longitud oste y $01^{\circ} 03$ ' 18 " de latitud sur. El sitio está a $73 \mathrm{msnm}$, la zona de vida es bh-T (Holdridge, 1987), con una precipitación promedio anual de $1537 \mathrm{~mm}$. La temperatura media diaria es de $24,2^{\circ} \mathrm{C}$, la humedad relativa es de $77.4 \%$, la heliofanía es de 823 horas luz año ${ }^{-1}$.

El estudio se realizó desde junio a octubre del 2006. Para fernansánchez y laurel se utilizó un diseño de bloques al azar con arreglo bifactorial. Es decir, se evaluó el comportamiento de dos factores: el factor (a), fueron los dos métodos de secado (caballete y cuadrado horizontal); y el factor (b), las tres secciones del fuste del árbol (basal: 0-4m, media: 4.1-8m y terminal: 8.1-12m). Por consiguiente resultaron seis tratamientos (T1: tablas de la sección basal apiladas en caballetes; T2: tablas de la sección media apiladas en caballetes; T3: tablas de la sección terminal apiladas en caballetes; T4: tablas de la sección basal apiladas en cuadrados horizontales; T5: tablas de la sección media apiladas en cuadrados hori- zontales; T6: tablas de la sección terminal apiladas en cuadrados horizontales) y se utilizaron tres repeticiones por tratamiento. Las unidades experimentales fueron tablas de fernansánchez y laurel de $120 \mathrm{~cm}$ de longitud, $15 \mathrm{~cm}$ de ancho, y $2.5 \mathrm{~cm}$ de espesor. Para el registro del contenido de humedad se seleccionó probetas de $10 \mathrm{~cm}$ x $2.5 \mathrm{~cm}$ x $2.5 \mathrm{~cm}$, tomadas de tablas de fernansánchez y laurel. El registro de datos se realizó una vez por mes. Las variables que se evaluaron, fueron: abarquillado, combado, torcedura, rajaduras, grietas superficiales y contenido de humedad. Para el análisis de datos se utilizó el programa informático SAS (Statistical Analysis System, 1999). Para la comparación entre los promedios de los tratamientos, se empleó la prueba de rangos múltiples de Tukey $(\mathrm{P} \leq 0.05)$.

Para el registro de los alabeos se colocaron las tablas sobre una superficie plana y luego con una regla graduada en centímetros se tomó la altura de las curvaturas. Para el registro de rajaduras y grietas superficiales, se tomó la longitud de las rajas y grietas, y se consideró un ancho de $2 \mathrm{~cm}$ para cada una; después se calculó el área de rajadura para establecer un porcentaje con respecto al área $\left(1800 \mathrm{~cm}^{2}\right.$, considerando una sola cara) de cada tabla evaluada. Para el registro del contenido de humedad se secaron las probetas en estufa cada vez durante 24 horas, a una temperatura de $103 \pm 2{ }^{\circ} \mathrm{C}$.

La metodología está basada en un estudio similar realizado por Orbe (1986) donde se evaluaron: combados, torceduras, rajaduras grietas superficiales y contenido de humedad en siete maderas del trópico ecuatoriano.

\section{Resultados}

\section{Experimento 1: T. cumingiana Fish. Mey}

El efecto simple de los métodos de secado sobre abarquillados, combados, torceduras, rajaduras, grietas superficiales y contenido de humedad no presentó diferencias significativas al secar a la intemperie tablas de fernansánchez mediante apilamientos en caballetes y cuadrados horizontales (Cuadro 1).

El efecto simple de las secciones del árbol sobre abarquillados, combados, torceduras, rajaduras, grietas superficiales y contenido de humedad, indicó que no hubieron diferencias significativas al secar tablas de las secciones basal, media y terminal de árboles de fernansánchez (Cuadro 2). 
Cuadro 1. Efecto simple de los métodos de secado sobre alteraciones, defectos y contenido de humedad en tablas de T. guayaquilensis. Finca "Rosita", octubre 2006

\begin{tabular}{lcccccc}
\hline & \multicolumn{6}{c}{ Variables } \\
\cline { 2 - 7 } Métodos de secado & $\begin{array}{c}\text { Abarquillados } \\
(\mathbf{m m})\end{array}$ & $\begin{array}{c}\text { Combados } \\
(\mathbf{m m})\end{array}$ & $\begin{array}{c}\text { Torceduras } \\
(\mathbf{m m})\end{array}$ & $\begin{array}{c}\text { Rajaduras } \\
\mathbf{( \% )}\end{array}$ & $\begin{array}{c}\text { Grietas } \\
\mathbf{( \% )}\end{array}$ & $\begin{array}{c}\text { Contenido de } \\
\text { humedad (\%) }\end{array}$ \\
\hline Caballetes & $2.11 \mathrm{a}$ & $4.17 \mathrm{a}$ & $4.67 \mathrm{a}$ & $0.81 \mathrm{a}$ & $0.00 \mathrm{a}$ & $15.10 \mathrm{a}$ \\
Cuadrados horizontales & $3.06 \mathrm{a}$ & $4.39 \mathrm{a}$ & $3.78 \mathrm{a}$ & $1.71 \mathrm{a}$ & $1.65 \mathrm{a}$ & $15.24 \mathrm{a}$ \\
\hline
\end{tabular}

Letras iguales no difieren estadísticamente según Tukey $(\mathrm{p} \leq 0.05)$

Cuadro 2. Efecto simple de las secciones del árbol sobre alteraciones, defectos y contenido de humedad en tablas de T. guayaquilensis. Finca " Rosita “, octubre 2006

\begin{tabular}{lcccccc}
\hline \multirow{2}{*}{$\begin{array}{c}\text { Secciones del } \\
\text { árbol }\end{array}$} & $\begin{array}{c}\text { Abarquillados } \\
(\mathbf{m m})\end{array}$ & $\begin{array}{c}\text { Combados } \\
\mathbf{( m m )}\end{array}$ & $\begin{array}{c}\text { Torceduras } \\
\mathbf{( m m )}\end{array}$ & $\begin{array}{c}\text { Rajaduras } \\
\mathbf{( \% )}\end{array}$ & $\begin{array}{c}\text { Grietas } \\
\mathbf{( \% )}\end{array}$ & $\begin{array}{c}\text { Contenido de } \\
\text { humedad } \mathbf{( \% )}\end{array}$ \\
\hline Basal & $2.00 \mathrm{a}$ & $4.33 \mathrm{a}$ & $4.58 \mathrm{a}$ & $1.03 \mathrm{a}$ & $0.00 \mathrm{a}$ & $15.65 \mathrm{a}$ \\
Media & $1.83 \mathrm{a}$ & $3.58 \mathrm{a}$ & $4.25 \mathrm{a}$ & $1.27 \mathrm{a}$ & $1.49 \mathrm{a}$ & $15.69 \mathrm{a}$ \\
Terminal & $2.67 \mathrm{a}$ & $4.92 \mathrm{a}$ & $3.83 \mathrm{a}$ & $1.49 \mathrm{a}$ & $0.99 \mathrm{a}$ & $14.16 \mathrm{a}$ \\
\hline
\end{tabular}

Letras iguales no difieren estadísticamente según Tukey $(\mathrm{p} \leq 0.05)$

La interacción entre métodos de secado y secciones del árbol no fue significativa para ninguna de las variables evaluadas.

grietas superficiales y contenido de humedad, indicó que no hubieron diferencias significativas al secar a la intemperie, tablas de laurel apiladas en caballetes y cuadrados horizontales (Cuadro 3).

El efecto simple de los métodos de secado
sobre abarquillados, combados, torceduras, rajaduras,

Cuadro 3. Efecto simple de los métodos de secado sobre alteraciones, defectos y contenido de humedad en tablas de C. alliodora. Finca " Rosita ", octubre 2006

\begin{tabular}{lcccccc}
\hline \multirow{2}{*}{$\begin{array}{c}\text { Métodos de } \\
\text { secado }\end{array}$} & $\begin{array}{c}\text { Compados } \\
\text { (mm) }\end{array}$ & $\begin{array}{c}\text { Combillados } \\
(\mathbf{m m})\end{array}$ & $\begin{array}{c}\text { Torceduras } \\
(\mathbf{m m})\end{array}$ & $\begin{array}{c}\text { Rajaduras } \\
\mathbf{( \% )}\end{array}$ & $\begin{array}{c}\text { Grietas } \\
(\%)\end{array}$ & $\begin{array}{c}\text { Contenido de } \\
\text { humedad (\%) }\end{array}$ \\
\hline Caballetes & $2.11 \mathrm{a}$ & $6.72 \mathrm{a}$ & $3.56 \mathrm{a}$ & $2.17 \mathrm{a}$ & $0.23 \mathrm{a}$ & $15.68 \mathrm{a}$ \\
$\begin{array}{l}\text { Cuadrados } \\
\text { horizontales }\end{array}$ & $3.06 \mathrm{a}$ & $5.44 \mathrm{a}$ & $3.28 \mathrm{a}$ & $3.00 \mathrm{a}$ & $1.51 \mathrm{a}$ & $15.48 \mathrm{a}$ \\
\hline
\end{tabular}

Letras iguales no difieren estadísticamente según Tukey $(\mathrm{p} \leq 0.05)$ 
El efecto simple de las secciones del árbol sobre abarquillados, combados, torceduras, rajaduras, grietas superficiales y contenido de humedad, indicó que no hubieron diferencias significativas al secar tablas de las secciones basal, media y terminal de árboles de laurel (Cuadro 4).

Cuadro 4. Efecto simple de las secciones del árbol sobre alteraciones, defectos y contenido de humedad en tablas de C. alliodora. Finca " Rosita ", octubre 2006

\begin{tabular}{lcccccc}
\hline \multirow{2}{*}{$\begin{array}{c}\text { Secciones del } \\
\text { árbol }\end{array}$} & $\begin{array}{c}\text { Abarquillados } \\
(\mathbf{m m})\end{array}$ & $\begin{array}{c}\text { Combados } \\
\mathbf{( m m )}\end{array}$ & $\begin{array}{c}\text { Torceduras } \\
\mathbf{( m m )}\end{array}$ & $\begin{array}{c}\text { Rajaduras } \\
(\mathbf{\%})\end{array}$ & $\begin{array}{c}\text { Grietas } \\
(\%)\end{array}$ & $\begin{array}{c}\text { Contenido de } \\
\text { humedad (\%) }\end{array}$ \\
\hline Basal & $2.08 \mathrm{a}$ & $5.25 \mathrm{a}$ & $2.50 \mathrm{a}$ & $2.81 \mathrm{a}$ & $0.43 \mathrm{a}$ & $15.84 \mathrm{a}$ \\
Media & $3.50 \mathrm{a}$ & $7.00 \mathrm{a}$ & $3.17 \mathrm{a}$ & $2.98 \mathrm{a}$ & $1.64 \mathrm{a}$ & $16.05 \mathrm{a}$ \\
Terminal & $2.17 \mathrm{a}$ & $6.00 \mathrm{a}$ & $4.58 \mathrm{a}$ & $1.97 \mathrm{a}$ & $0.55 \mathrm{a}$ & $14.86 \mathrm{a}$ \\
\hline
\end{tabular}

Letras iguales no difieren estadísticamente según Tukey $(\mathrm{p} \leq 0.05)$

La interacción entre métodos de secado y secciones del árbol no fue significativa para ninguna de las variables evaluadas.

\section{Discusión}

$\mathrm{E}$ n la disposición de cuadrados horizontales, el combado en fernansánchez fue de $4.39 \mathrm{~mm}$ y el laurel presentó $5.44 \mathrm{~mm}$. En el apilado en caballetes, este alabeo fue de $4.17 \mathrm{~mm}$ en fernansánchez y $6.72 \mathrm{~mm}$ en laurel. Estos valores fueron superiores a los indicados en otras especies por Orbe (1981), quien puntualiza que Vochysia guianensis (bella maría blanca) presentó 0.89 $\mathrm{mm}$, Nectandra reticulata (canelón blanco) $0.38 \mathrm{~mm}$ y Nectandra sp. (jigua) $0.90 \mathrm{~mm}$; mientras que Orbe (1986), determinó para Cedrelinga catenaeformes (seique) $0.51 \mathrm{~mm}$ y Nectandra sp. (canelo) $0.98 \mathrm{~mm}$.

Con respecto a la torcedura, el fernansánchez presentó $3.78 \mathrm{~mm}$ y el laurel $3.28 \mathrm{~mm}$ mediante el apilado en cuadrados horizontales. En lo referente al apilado en caballetes, el fernansánchez presentó $4.67 \mathrm{~mm}$ y el laurel $3.56 \mathrm{~mm}$. Estos valores fueron superiores a los señalados por Orbe (1981), quien establece que Vochysia guianensis (bella maría blanca) presentó $2.38 \mathrm{~mm}$, Nectandra reticulata (canelón blanco) $1.44 \mathrm{~mm}$ y Nectandra sp. (jigua) $1.14 \mathrm{~mm}$; mientras que Orbe (1986), determinó para Cedrelinga catenaeformis (seique) 1.67 mm y Nectandra $s p$. (canelo) $2.53 \mathrm{~mm}$.

La rajadura en fernansánchez fue de $1.71 \%$ y el laurel presentó $3 \%$ en la disposición de cuadrados horizontales. En cambio en el apilado en caballetes, este defecto fue de $0.81 \%$ en fernansánchez y en laurel $2.17 \%$. Estos valores fueron superiores a los indicados por Orbe (1981) quien indica que Vochysia guianensis (bella maría blanca) presentó $0.42 \%$, Nectandra reticulata (canelón blanco) $0.15 \%$ y Nectandra sp. (jigua) $0.35 \%$; mientras que Orbe (1986), determinó para $\mathrm{Ce}$ drelinga catenaeformis (seique) que no presentó rajaduras; el Nectandra sp. (canelo) superó al fernansánchez puesto que mostró $2.84 \%$ de rajadura.

En lo concerniente a grietas superficiales, se observó que el fernansánchez presentó $1.65 \%$ y el laurel $1.51 \%$ mediante el apilado en cuadrados horizontales; en lo concerniente al apilado en caballetes, el fernansánchez no presentó grietas y el laurel $0.23 \%$; en cambio Orbe (1981) estableció que Vochysia guianensis (bella maría blanca) no presentó este defecto, lo mismo ocurrió con Nectandra reticulata (canelón blanco) y Nectandra sp. (jigua); mientras que Orbe (1986), determinó que el Cedrelinga catenaeformis (seique) tampoco presentó grietas pero el Nectandra sp. (canelo) superó al laurel ya que presentó $1.53 \%$.

Si se considera las condiciones climáticas del sitio donde se llevó a cabo el ensayo, se deduce que la humedad relativa promedio $(84 \%)$ de los meses en que se realizó el experimento, fue probablemente uno de los factores determinantes para que sea relativamente largo el tiempo (90 días) para que el fernansánchez y laurel bajen su alto contenido de humedad hasta alcanzar un contenido de humedad de equilibrio (CH: $18-19 \%)$.

\section{Conclusión}

Con base en los resultados obtenidos en el presente estudio se concluye que el fernansánchez se deforma menos que el laurel al secarse a la intemperie, pero ambas especies se pueden apilar indistintamente mediante caballetes o cuadrados horizontales. 


\section{LITERATURA CITADA}

COPANT. 1969. Glosario de Maderas. Buenos Aires Argentina. Comisión Panamericana de Normas Técnicas (COPANT). 20 p.

Fernández, J. 1998. Manual de Secado de Maderas. Québec - Canadá. Centro de Investigación Forestal (CIFOR - INIA). Asociación de Investigación Técnica de las Industrias de la Madera y Corcho. $152 \mathrm{p}$.

Holdridge, L. 1987. Ecología basada en las zonas de vida. San José, Costa Rica, IICA. 216 p.

Junta del Acuerdo de Cartagena. 1981. Descripción General y Anatómica de 105 Maderas del Grupo Andino. Cali - Colombia. Editorial Carvajal S. A. $441 \mathrm{p}$.

Junta del Acuerdo de Cartagena. 1989. Manual del Grupo Andino para el Secado de Maderas. Cali - Colombia. Editorial Carvajal S. A. 124 p.

OIMT. 2001. Guías Técnicas para el Establecimiento y Manejo de Plantaciones Forestales Productivas en el Litoral Ecuatoriano. Proyecto Piloto para la Reforestación y Rehabilitación de Tierras Forestales Degradadas en El Ecuador. CORMADERA. Quito - Ecuador. 179 p.
Orbe, J. 1986. Estudio de Secado de Siete Maderas del Trópico Ecuatoriano. Conocoto-Ecuador. Ministerio de Agricultura y Ganadería. Centro de Capacitación e Investigación Forestal "Luciano Andrade Marín”. 18 p.

Orbe, J. 1989. Contenido de humedad de equilibrio de la madera en varias localidades del Ecuador. Conocoto - Ecuador. Ministerio de Agricultura y Ganadería. Centro de Capacitación e Investigación Forestal "Luciano Andrade Marín”. 48 p.

Peña, S. 2000. Tecnología de la Madera. Madrid - España. Ministerio de Agricultura, Pesca y Alimentación. Universidad Politécnica de Madrid (ETSIM). 651 p.

SAS. 1999. SAS. User's guide: Statistics [CD-ROM Computer file]. Versión 8. Statistical Analysis Sistem Institute. Cary, NC. 